International Journal of Pure and Applied Mathematics

Volume 114 No. 3 2017, 571-582

ISSN: 1311-8080 (printed version); ISSN: 1314-3395 (on-line version)

url: http://www.ijpam.eu

doi: 10.12732 /ijpam.v114i3.12

\title{
MHD COUETTE FLOW IN AN ANNULAR POROUS REGION BETWEEN TWO COAXIAL CYLINDERS
}

\author{
Vineet Kumar Verma ${ }^{1}$, Pawan Kumar Dixit ${ }^{2} \S$ \\ ${ }^{1,2}$ Department of Mathematics and Astronomy \\ University of Lucknow \\ Lucknow, INDIA
}

\begin{abstract}
This paper deals with the magnetohydrodynamic laminar steady flow of a viscous incompressible fluid in an annular porous region between two coaxial cylindrical pipes under the uniform transverse magnetic field. The outer pipe is moving with uniform velocity along the axis of pipe and inner is fixed. Flow within the porous region is governed by the Brinkman equation. Analytical expressions for velocity, volume flow rate, average velocity and stress on the walls of pipes are obtained. The effect of various parameters such as permeability parameter, Hartmaan number and pressure gradient on the flow are discussed and obtained results are exhibited graphically.
\end{abstract}

AMS Subject Classification: 76SXX, 76WXX

Key Words: porous medium, Brinkman equation, couette flow, MHD Flow, Hartmann number, permeability parameter

\section{Introduction}

Convection in porous media is important in a variety of practical situations, including thermal insulation, oil and gas extraction, ground water movement, packed bed reactors, etc. Flow in a porous channel of different shapes specially parallel plates and cylindrical pipes has been extensively investigated due to its numerous applications in geophysics, chemical engineering, and petroleum industry etc. The effect of magnetic field on the flow becomes important

Received: February 2, 2017

Revised: $\quad$ May 10, 2017

Published: $\quad$ May 23, 2017

(c) 2017 Academic Publications, Ltd. url: www.acadpubl.eu

$\S$ Correspondence author 
when the flowing fluid is conducting fluid. Many authors dealt with the flow through porous channels namely, Kaviany (1985), Parang and Keyhani (1987), Nakayama et al. (1988), Vafai and Kim (1989), Chikh (1995), Nield et al. (1996), Nield and Kuznetsov (2000), Haji-Sheikh and Vafai (2004), Hooman and Ranjbar-Kani (2004), Hooman (2006, 2007), Pantokratoras and Fang (2009), Wang (2008, 2010, 2011), Zhao et.al. (2010), Ng and Wang (2010), Verma and Datta (2012a,b), Verma (2014), Verma and Singh (2014, 2015) etc.

An important class of problems related to flow of electrically conducting fluid in porous medium is MHD flow. There is huge literature available on the MHD flow of clear fluid but we have very little literature on the fluid flow through porous channels. Few author have dealt problem of this kind such as Krishna et.al.(2002) investigated an initial value investigation of the hydromagnetic convection of a viscous electrically conducting fluid through a porous medium in a rotating parallel plate channel. Khan et al. (2008) found the analytic solution for flow of a magnetohydrodynamic Sisko fluid through a porous medium by introducing the modified Darcys law using the homotopy analysis method. In the limiting case, the obtained solution reduces to the well-known solutions for a Newtonian fluid in nonporous and porous media. Hayat et al. (2008) investigated the magnetohydrodynamic flow of a non-Newtonian fluid filling the porous space in a channel with compliant walls. They used constitutive equations of a Jeffery fluid. The flow in the considered problem was created due to sinusoidal traveling waves on the channel walls. The resulting problem was solved analytically. Makinde and Mhone (2009) presented a linear stability analysis to trace the time evolution of an infinitesimal, two-dimensional disturbance imposed on the base flow of an electrically conducting fluid in a channel filled with a saturated porous medium under the influence of a transversely imposed magnetic field. Pantokratoras and Fang (2010) investigate the Poiseuille and Couette flow in a fluid-saturated Darcy-Brinkman porous medium channel with an electrically conducting fluid under the action of a magnetic and electric field. Exact analytical solutions are derived for fluid velocity. Zhao et al. (2010) extend the previous work Pantokratoras and Fang (2010) to the case with a Darcy-Brinkman-Forchheimer porous medium. Srivastava et.al.(2013) Poiseuille and Couette flow of an electrically conducting fluid through a porous medium of variable permeability under the transverse magnetic field. They used the Brinkman equation for flow through the porous medium and obtained a numerical solution for velocity and the volumetric flow rate using the Galerkin method.

In the present problem we have studied the steady and laminar flow of a 
viscous, incompressible fluid in an annular coaxial translating cylinders filled saturated porous medium under the uniform transverse magnetic field. Exact solution is obtained. The Brinkman equation (1947) is used for flow through a porous medium. Flow characteristics such as velocity, rate of volume flow, average velocity and shearing stress are obtained and exhibited graphically. The effect of various parameters has been analysed. it has been shown that effect of permeability parameter and Hartmann number has strong effect on flow characteristics. The problem is classical in the field of flow through porous media and new in literature.

\section{Mathematical formulation}

We consider steady flow of viscous fluid in annular region of two coaxial translating cylinders which is filled with a porous medium under the uniform transversely applied magnetic field. Outer cylindrical pipe is translating with uniform velocity $U$ parallel to the axis and inner is stationary. we use cylindrical coordinate system $\left(r^{*}, \theta, z^{*}\right)$. There is unidirectional flow along $z^{*}$ direction inside an annular region. We assume radius of outer and inner pipe is $R_{2}$ and $R_{1}$, respectively. The applied constant pressure gradient is $\frac{\partial p^{*}}{\partial z^{*}}$. The flow within annular porous region is governed by Brinkman (1947) equation, which is in the presence of applied magnetic field is given by

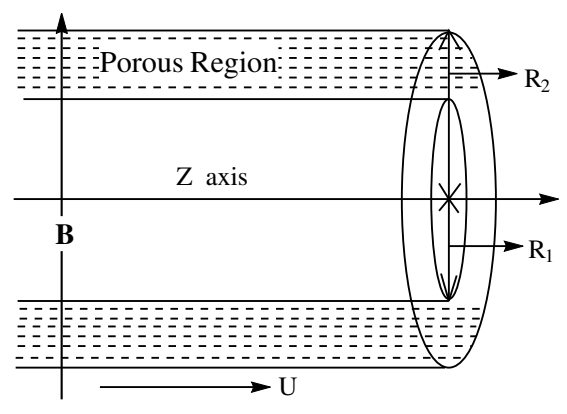

Fig. 1: Schematic diagram of the problem.

$$
\mu_{e}\left(\frac{d^{2} u^{*}}{d r^{* 2}}+\frac{1}{r^{*}} \frac{d u^{*}}{d r^{*}}\right)-\frac{\mu}{k} u^{*}-\sigma_{o} B^{2} u^{*}=\frac{\partial p^{*}}{\partial z^{*}}
$$

where $u^{*}$ is the fluid velocity, $\mu_{e}$ is the effective viscosity, $\mu$ is the fluid viscosity, $k$ is the permeability of porous medium, $\sigma_{o}$ is the electrical conductivity of 
fluid and $B$ is the magnetic field. Here we assume that the magnetic Reynolds number is small and there is no external electric field. Therefore, induced current can be neglected and internal causes, such as separation of charges or polarization, do not rise to induced electric field. In this case, the Lorentz force acting on the fluid will be $\sigma_{o} B^{2} u^{*}$ and is opposite to the direction of flow. Authors have different opinion about effective viscosity. According to Liu and Masliyah (2005) depending on the type of porous medium effective viscosity may be greater or smaller than fluid viscosity. Many Author, for example Brinkman (1947) and chikh et al. (1995) assume $\mu_{e}=\mu$, this is valid for porous medium of high porosity. We also assume $\mu_{e}=\mu$. With this assumption, Brinkman momentum eq.(1) becomes

$$
\frac{d^{2} u^{*}}{d r^{*}}+\frac{1}{r^{*}} \frac{d u^{*}}{d r^{*}}-\frac{u^{*}}{k}-\frac{\sigma_{0} B^{2} u^{*}}{\mu}=\frac{1}{\mu} \frac{\partial p^{*}}{\partial z^{*}}
$$

Boundary conditions for the flow are no slip condition on the surface of inner and outer cylinder. That are

$$
u^{*}=0 \quad \text { at } \quad r^{*}=R_{1} \quad \text { and } \quad u^{*}=U \text { at } r^{*}=R_{2}
$$

We introduce non dimensional quantities as follows

$$
r=\frac{r^{*}}{R_{1}}, \quad u=\frac{u^{*}}{U}
$$

Boundary conditions (3) in terms of non dimensional quantities are

$$
u(1)=0 \quad \text { and } \quad u(q)=1
$$

Here $q=R_{2} / R_{1}$ is defined as gap parameter. Eq.(2) in non dimensional form is

$$
\frac{d^{2} u}{d r^{2}}+\frac{1}{r} \frac{d u}{d r}-\beta^{2} u=-P
$$

where $\beta=\sqrt{\sigma^{2}+M^{2}} \cdot \sigma^{2}=R_{1}^{2} / k$ is defined as permeability parameter, $M=$ $B \sqrt{\frac{\sigma_{0}}{\mu}}$ is the Hartmaan number and $P=-\frac{R_{1}^{2}}{\mu U} \frac{\partial p^{*}}{\partial z^{*}}$ is pressure gradient. All these quantities are non-dimensional.

\section{Solution}

Equation (6) can be written as

$$
r^{2} \frac{d^{2} u}{d r^{2}}+r \frac{d u}{d r}-\beta^{2} r^{2} u=-r^{2} P
$$


Eq.(7) is a modified Bessel's equation of order zero. Its general solution is given by

$$
u(r)=a_{1} I_{o}(\beta r)+a_{2} K_{o}(\beta r)+\frac{P}{\beta^{2}}
$$

where $I_{o}$ and $K_{o}$ are the modified Bessel functions of zeroth order of first and second kind, respectively. $a_{1}$ and $a_{2}$ are constants of integration. Using the boundary conditions (5), we get

$$
\begin{aligned}
a_{1} & =\frac{\beta^{2} K_{o}(\beta)+P\left(K_{o}(\beta q)-K_{o}(\beta)\right)}{\beta^{2}\left(I_{o}(\beta q) K_{o}(\beta)-I_{o}(\beta) K_{o}(\beta q)\right)}, \\
a_{2} & =\frac{\beta^{2} I_{o}(\beta)+P\left(I_{o}(\beta q)-I_{o}(\beta)\right)}{\beta^{2}\left(I_{o}(\beta) K_{o}(\beta q)-I_{o}(\beta q) K_{o}(\beta)\right)}
\end{aligned}
$$

With this value of constants $a_{1}$ and $a_{2}$ velocity of flow within annular porous region is given by eq.(8). In limiting case, when permeability of the porous medium is infinite and there is no magnetic field. i.e. when $\beta=\sqrt{\sigma^{2}+M^{2}} \rightarrow$ 0. Eq.(8) gives us the velocity $u_{0}$ for clear fluid flow

$$
u_{0}=\frac{P\left(1-r^{2}\right) \log q+\left(P\left(q^{2}-1\right)+4\right) \log r}{4 \log q}
$$

which is the classical results of couette flow in an annular cylinder.

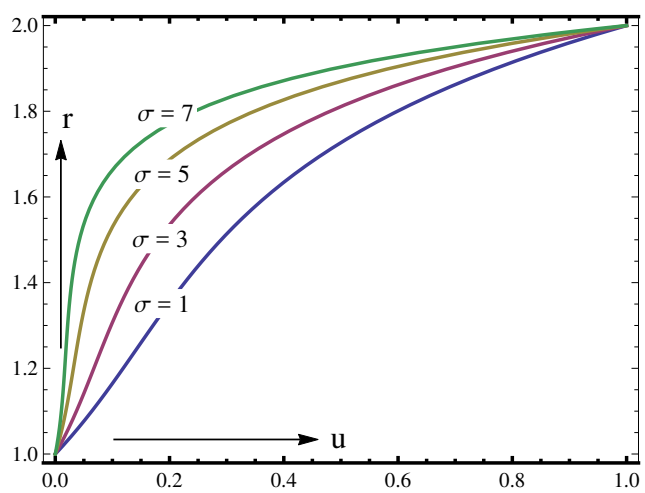

Fig. 2: Velocity profiles for different $\sigma$ when $\mathrm{q}=2, \mathrm{P}=1$ and $M=3$.

\subsection{Rate of volume flow}

The dimensionless rate of volume flow through the cross-section of annular tube is given by 


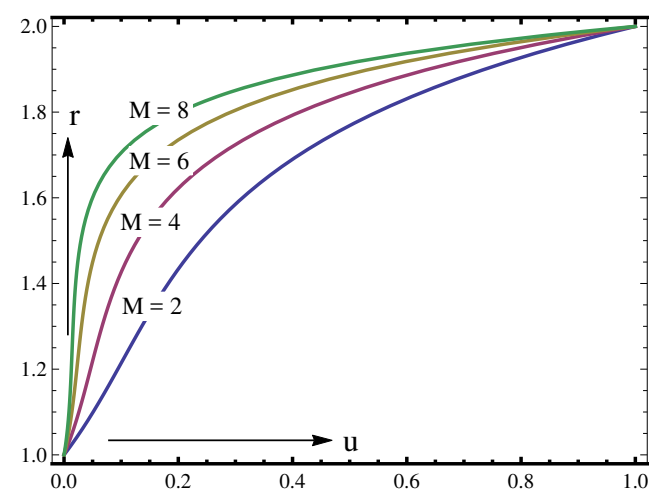

Fig. 3: Velocity profiles for different $M$ when $\mathrm{q}=2, \sigma=3$ and $P=1$.

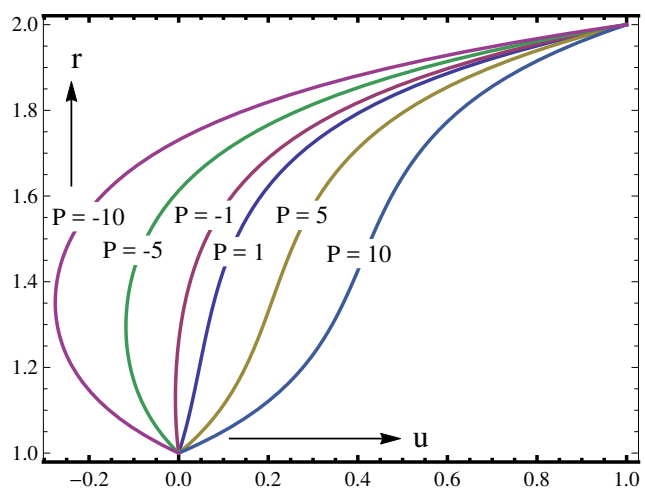

Fig. 4: Velocity profiles for different $\mathrm{P}$ when $\mathrm{q}=2, \mathrm{M}=4$ and $\sigma=3$. 


$$
Q=2 \pi \int_{1}^{q} u(r) r d r
$$

Substituting $u$ from eq.(8), after integration one obtains

$$
Q=2 \pi\left[\begin{array}{c}
\left(\frac{P\left(q^{2}-1\right)}{2 \beta^{2}}\right)+a_{1}\left(\frac{q I_{1}(\beta q)-I_{1}(\beta)}{\beta}\right) \\
+a_{2}\left(\frac{K_{1}(\beta)-q K_{1}(\beta q)}{\beta}\right)
\end{array}\right]
$$

where $I_{1}$ and $K_{1}$ are the modified Bessel function of first order and $a_{1}, a_{2}$ are given by eq.(9). In the evaluation of above integrals the following identity [Ref. Abramowitz and Stegun (1970)] has been used

$$
\left(\frac{1}{z} \frac{d}{d z}\right)^{m}\left\{z^{\nu} £_{\nu}(z)\right\}=z^{\nu-m} £_{\nu-m}(z)
$$

with $m=1$ and $\nu=1$. $£_{\nu}$ denotes $I_{\nu}$ and $e^{\nu \pi i} K_{\nu}$.

The dimensionless volume flow rate $Q_{o}$ for clear fluid flow in absence of magnetic field is obtained by taking limit $\beta \rightarrow 0$ in eq.(12) or by direct evaluation of integral (10) with $u=u_{o}$, which is

$$
Q_{0}=\frac{\pi}{8}\left[P\left(q^{4}-1\right)+8 q^{2}-\frac{\left(q^{2}-1\right)\left(P\left(q^{2}-1\right)+4\right)}{\log q}\right]
$$

which is the classical results of couette flow in an annular cylinder.

\subsection{Average velocity}

The dimensionless average velocity of the flow is defined as

$$
u_{\text {avg }}=\frac{Q}{\pi\left(q^{2}-1\right)}
$$

Substituting $Q$ from the eq.(12) in the above equation, we obtain average velocity of the flow as

$$
u_{\text {avg }}=\frac{2}{\left(q^{2}-1\right)}\left[\begin{array}{c}
\left(\frac{P\left(q^{2}-1\right)}{2 \beta^{2}}\right)+a_{1}\left(\frac{q I_{1}(\beta q)-I_{1}(\beta)}{\beta}\right) \\
+a_{2}\left(\frac{K_{1}(\beta)-q K_{1}(\beta q)}{\beta}\right)
\end{array}\right]
$$

For clear fluid flow in absence of magnetic field i.e. when $(\beta \rightarrow 0$ the average velocity of the flow is obtained as

$$
\left(u_{\text {avg }}\right)_{\beta=0}=\frac{1}{8}\left[P\left(q^{2}+1\right)+\frac{8 q^{2}}{\left(q^{2}-1\right)}-\frac{\left(P\left(q^{2}-1\right)+4\right)}{\log q}\right]
$$




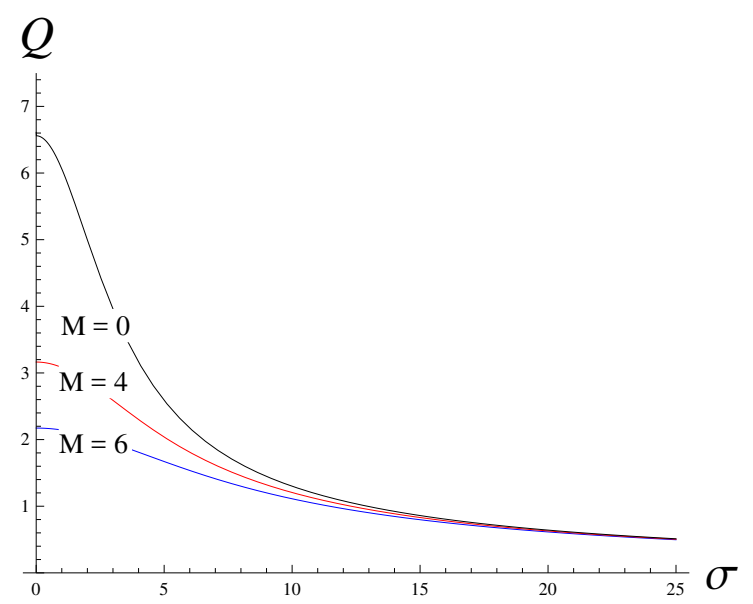

Fig. 5: Variation of flow rate with $\sigma$ for different $\mathrm{M}$ when $\mathrm{P}=1$ and $\mathrm{q}=2$.

\subsection{Shearing stress}

The dimensionless shearing stress at any point is given by

$$
\tau_{r z}(r)=\frac{d u}{d r}
$$

Substituting $u$ from eq.(8) and differentiating the modified Bessel functions $I_{o}(\beta r)$ and $K_{o}(\beta r)$ with use of the identity $\frac{d}{d r} I_{o}(r)=I_{1}(r)$ and $\frac{d}{d r} K_{o}(r)=$ $-K_{1}(r)$ [Ref. Abramowitz and Stegun (1970)], one obtains

$$
\tau_{r z}(r)=a_{1} \beta I_{1}(\beta r)-a_{2} \beta K_{1}(\beta r)
$$

where $I_{1}$ and $K_{1}$ are modified Bessel function of order one. Stress on the surface of inner and outer cylinder is obtained by putting $r=1$ and $r=q$ in eq.(19) respectively and using the appropriate sign. Thus, providing

$$
\begin{gathered}
\tau_{r z}(1)=a_{1} \beta I_{1}(\beta)-a_{2} \beta K_{1}(\beta) \\
\tau_{r z}(q)=a_{1} \beta I_{1}(\beta q)-a_{2} \beta K_{1}(\beta q)
\end{gathered}
$$

where $a_{1}, a_{2}$ are given by eq.(9). Dimensionless shearing stress on the surface of inner and outer cylinders for clear fluid flow and there is no magnetic field ( i.e. $\sigma \rightarrow 0$ and $M \rightarrow 0$ ) are obtained by taking limit $\beta \rightarrow 0$ in eq.(20) and eq. (21), respectively. Then

$$
\tau_{r z}(1)=\frac{P\left(q^{2}-1\right)+4-2 P \log q}{4 \log q}
$$




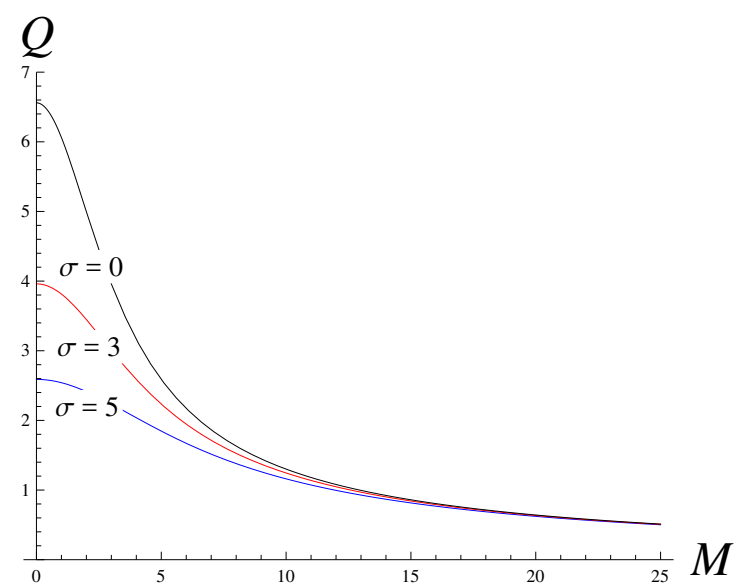

Fig. 6: Variation of flow rate with $\mathrm{M}$ for different $\sigma$ when $\mathrm{P}=1$ and $\mathrm{q}=2$.

$$
\tau_{r z}(q)=\frac{P\left(q^{2}-1\right)+4-2 P q^{2} \log q}{4 q \log q}
$$

which is the classical results of couette flow in an annular cylinder.

\section{Results and Discussion}

The influence of permeability parameter $\sigma=R_{1} / \sqrt{k}$ on the flow velocity for fixed $M=3, P=1$ and $q=2$ is depicted in the fig.(2). From the figure we can observe that the velocity is showing increasing behavior with the decrement in the value of $\sigma$. This is because permeability $k$ increases as $\sigma$ decreases.

The effect of Hartmann number $M$ on the flow velocity for fixed $\sigma=3, P=$ 1 and $q=2$ is shown in the fig.(3). Figure shows that the velocity decreases with increase in Hartmann number. This is because of the fact that increase in magnetic field increases the Lorentz force in opposite to the direction of flow. So we can use magnetic field to control the fluid velocity.

In fig.(4) the effect of pressure gradient $P$ on the velocity is shown for fixed values of $\sigma=3, M=4$ and gap parameter $q=2$. Figure shows that the velocity increases when the values of $P$ grow.

In fig.(5) variation of volume flow rate $Q$ with permeability parameter $\sigma$ for fixed value $M=3, P=1$ is shown for different values $\sigma$ and when $q=2$. We observe that volume flow rate $Q$ decreases with increment in $\sigma$ in annular region of coaxial cylinders. This is because increase in $\sigma$ means decrease in the permeability of medium. 
In fig.(6) represents the effect of Hartmann number $M$ (magnetic field) on the flow for fixed value of $\sigma$. We observe that volume flow rate $Q$ decreases with increment in $M$ in annular region of coaxial cylinders. This is because of the fact that increase in magnetic field increases the Lorentz force in opposite to the direction of flow.

\section{Conclusion}

An analytical solution of laminar flow of a viscous incompressible conducting fluid in an annular region between two coaxial translating cylindrical tubes filled with a porous medium is obtained under the uniform transverse magnetic field. The analytical expression for the velocity, flow rate, average velocity and shear stress are obtained by using Brinkman equation. The effect of various parameters on the flow characteristics are discussed and obtained results are exhibited graphically. In limiting case when magnetic field and permeability is zero the obtained results reduces to the classical results of couette flow in an annular cylinder. We found that magnetic field and permeability parameter have remarkable effect of the flow.

\section{References}

[1] Abramowitz and Stegun : A hand book of mathematical functions, Wiley-Interscience, NewYork, (1972), 557-558.

[2] M. Kaviany, Laminar flow through a porous channel bounded by isothermal parallel plates, Int. J. Heat Mass Transfer, vol. 28(1985), pp. 851-858.

[3] M. Parang and M. Keyhani, Boundary effects in laminar mixed convection flow through an annular porous medium, ASME J. Heat Transfer, vol. 109(1987), pp. 1039-1041.

[4] A. Nakayama, H. Koyama and F. Kuwahara, An analysis on forced convection in a channel filled with a BrinkmanDarcy porous medium: Exact and approximate solutions, Warme Und Stoffubertragung, vol. 23(1988), pp. 291-295.

[5] K. Vafai and S. J. Kim, Forced convection in a channel filled with porous medium: An exact solution, ASME J. Heat Transfer, vol. 111(1989), pp. 1103-1106.

[6] S. Chikh, A. Boumedien, K. Bouhadef and G. Lauriat, Analytical solution of non-Darcian forced convection in an annular duct partially filled with a porous medium, Int. J. Heat Mass Transfer, vol. 38(1995), pp. 1543-1551.

[7] H. C. Brinkman, A calculation of the viscous force exerted by a flowing fluid on a dense swarm of particles, Appl.Sci.Res.,Vol. A1(1947), pp. 27-34.

[8] D. A. Nield, S. L. M. Junqueira, and J. L. Lage, Forced convection in a fluid-saturated porous medium channel with isothermal or isoflux boundaries, J. Fluid Mech., vol. 322(1996), pp. 201-214. 
[9] D. A. Nield and A. V. Kuznetsov, Effects of heterogeneity in forced convection in a porous medium: Parallel plate channel or circular duct, Int. J. Heat Mass Transfer, vol. 43(2000), pp. 4119- 4134.

[10] A. Haji-Sheikh and K. Vafai, Analysis of flow and heat transfer in porous media imbedded inside various shaped ducts, Int. J. Heat Mass Transfer, vol. 47(2004), pp. 1889-1905.

[11] K. Hooman, A perturbation solution for forced convection in a porous-saturated duct, $J$. Comput. Appl. Math., vol. 211(2006), pp. 57-66.

[12] K. Hooman and H. Gurgenci, A theoretical analysis of forced convection in a porous saturated circular tube: Brinkman- Forchheimer model, Transport Porous Media, vol. 69(2007), pp. 289-300.

[13] K. Hooman and A. A. Ranjbar-Kani, A perturbation based analysis toinvestigate forced convection in a porous saturated tube, J. Comput. Appl. Math., vol. 162(2004), pp. 411-419.

[14] A. Pantokratoras and T. Fang, Flow of a weakly conducting fluid in a channel filled with a porous medium, Transport Porous Media, vol. 83(2010), pp. 667-676.

[15] C. Y. Wang, Flow through super-elliptic ducts filled with a Darcy-Brinkman medium, Transp. Porous Media, vol. 81(2010), pp. 207-217.

[16] C. Y. Wang, Flow and Heat Transfer Through a Polygonal Duct filled with a Porous Medium, Transport porous media, vol. 90(2011), pp.321-332.

[17] C. Y. Wang, Analytical solution for forced convection in a semicircular channel filled with a porous medium, Transp. Porous Media, vol. 73(2008), pp. 369-378.

[18] B.Q. Zhao, A. Pantokratoras, T. G. Fang, and S.J. Liao, Flow of a Weakly Conducting Fluid in a Channel Filled with a Darcy-Brinkman-Forchheimer Porous Medium, Transport in Porous Media, vol. 85(2010), pp. 131-142.

[19] Ng, Chiu-On and C. Y. Wang, DarcyBrinkman Flow Through a Corrugated Channel, Transp Porous Med., vol. 85(2010), pp. 605-618.

[20] V. K. Verma and S. Datta, Flow in a channel filled by heterogeneous porous mediu with a linear permeability variation, Special Topics and Reviews in Porous Media An International Journal, vol. 3(3)(2012a), pp. $201-208$.

[21] V. K. Verma and S. Datta, Flow in an annular channel filled with a porous medium of variable permeability, Journal of Porous Media, vol. 15(10)(2012b), pp. 891 - 899.

[22] V. K. Verma, Analytical solution of magnetohydrodynamic flow with varying viscosity in an annular channel, Adv. Theor. Appl. Math., vol.9(2014a), pp. $105-122$.

[23] V. K. Verma and S. Singh, Flow between coaxial rotating cylinders filled by porous medium of variable permeability, Special Topics and Reviews in Porous Media An International Journal, vol.5(4)(2014b), pp. 355 - 359.

[24] V. K. Verma and S. Singh, Magnetohydrodynamic flow in a circular channel filled with a porous medium., Journal of Porous Media, vol.18(9)(2015), pp. 923 - 928.

[25] D. V. Krishna, D. R. V. Prasada Rao and A. S. R. Murthy, Hydromagnetic convection flow through a porous medium in a rotating channel. Journal of Engineering Physics and Thermophysics, Vol. 75(2)(2002).

[26] M. Khan, Z. Abbas and T. Hayat, Analytic solution for ow of Sisko uid through a porous medium, Transport in Porous Media, Volume 71(2008), 23-37. 
[27] T. Hayat, M. Javed and N. Ali, MHD Peristaltic Transport of a Jeffery Fluid in a Channel With Compliant Walls and Porous Space, Trans Porous Med. vol. 74(2008), 259-274.

[28] O. D. Makinde and P. Y. Mhone, On Temporal Stability Analysis for Hydromag- netic Flow in a Channel Filled with a Saturated Porous Medium. Flow Turbulence Combust. Vol. 83(2009), 21-32.

[29] B.G. Srivastava and Satya Deo, Effect of magnetic field on the viscous fluid fow in a channel filled with porous medium of variable permeability, Applied Mathematics and Computation, vol. 219(2013), 8959-8964. 